\title{
Biontechs Steuerbooster und seine Nebenwirkungen auf das Finanzgefüge
}

\begin{abstract}
Der Mainzer Impfstoffhersteller Biontech hatte 2021 eine glänzende Geschäftsentwicklung, die zu einem sprunghaften Anstieg der rheinland-pfälzischen Steuereinnahmen geführt hat. Damit gehört das seit jeher finanzschwache Land Rheinland-Pfalz plötzlich zu den finanzstärksten Bundesländern und zählt damit zu den Geberländern im Finanzausgleichssystem. Ein so starker Anstieg der Finanzkraft führt jedoch zu erheblichen fiskalischen Spillover-Effekten, die letztlich zu problematischen Verteilungsergebnissen führen können. Die fiskalischen Folgen der durch Biontech induzierten Steuererhöhung führen speziell für den rheinland-pfälzischen Landeshaushalt zu überraschenden Ergebnissen.
\end{abstract}

\begin{abstract}
Mit der Versendung der vorläufigen Abrechnung des bundesstaatlichen Finanzausgleichs 2021 des Bundesministeriums der Finanzen an die Länder (BMF, 2022a) wurde amtlich, was schon im Vorfeld kolportiert wurde (FM RLP, 2021). Rheinland-Pfalz verzeichnete in den letzten Monaten des Jahres 2021 ein außergewöhnlich hohes Steueraufkommen und findet sich erstmals auf der Geberseite des Finanzausgleichs wieder. Diese Mehreinnahmen sind allein bei zwei Steuerarten und jeweils in einem einzigen Monat aufgetreten. Im November 2021 vereinnahmten die rheinland-pfälzischen Steuerbehörden bei der Körperschaftsteuer fast 1,7 Mrd. Euro (BMF, 2022b). Dabei ist der November kein Vorauszahlungsmonat und erbringt im Regelfall nur ein sehr geringes, bzw. häufig sogar ein
\end{abstract}

(C) Der/die Autor:in 2022. Open Access: Dieser Artikel wird unter der Creative Commons Namensnennung 4.0 International Lizenz veröffentlicht (creativecommons.org/licenses/by/4.0/deed.de).

Open Access wird durch die ZBW - Leibniz-Informationszentrum Wirtschaft gefördert.

* Die Autoren vertreten ihre persönliche Meinung, nicht die der Institutionen, denen sie angehören. Die für diesen Beitrag verwendeten Daten beruhen auf öffentlich zugänglichen Statistiken und Presseangaben sowie aufbauend auf eigenen Schätzungen und Berechnungen.

Reinhold Weiß ist Referatsleiter im Hessischen

Ministerium der Finanzen.

Dr. Kristof Kanzler ist dort Referent.

Robert Fiesel ist Referent im Ministerium für Finanzen

Baden-Württemberg. negatives Aufkommen. ${ }^{1}$ Die Tragweite dieses Steuerfalls wird besonders deutlich, wenn man die Einnahmen des Landes Rheinland-Pfalz aus dieser Steuerart über einen längeren Zeitraum betrachtet. Abbildung 1 zeigt, dass 2021 das Aufkommensniveau der Vorjahre um mehr als das Doppelte übertroffen wurde.

Auch bei den nichtveranlagten Steuern vom Ertrag war im Dezember 2021 mit 1,4 Mrd. Euro ein ungewöhnlich hohes Monatsaufkommen zu verzeichnen (BMF, 2022b). Dieses war sogar höher als das Jahresaufkommen in den Vorjahren und führte damit für 2021 zu einem Aufkommen, das mit 2,6 Mrd. Euro ebenfalls weit über dem Durchschnitt der Vorjahre lag.

Ausschlaggebend für diese Entwicklung dürfte die fulminante Geschäftsentwicklung eines Mainzer Impfstoffherstellers sein, die der Stadt Mainz einen derart spektakulären Geldsegen verschafft, dass er sogar in der internationalen Presse Aufsehen erregte (The Guardian, 2021). Aufgrund weiterer Presseartikel lässt sich die Dimension dieses Steuerfalles zumindest bei der Gewerbesteuer und wegen des engen inhaltlichen Zusammenhangs damit auch bei der Körperschaftsteuer relativ genau eingrenzen (RND, 2021; Hessenschau, 2021). Wegen der bereits erfolgten Namensnennung des Impfstoffherstellers in den zuvor zitierten Artikeln und der Pressemitteilung des Ministeriums der Finanzen Rheinland-Pfalz (FM RLP, 2021) kann dieser Steuerfall auch als „Biontech-Fall“ bezeichnet werden.

Es erscheint zwar äußerst plausibel, dass auch das auBergewöhnlich hohe Aufkommen im Dezember bei den nichtveranlagten Steuern vom Ertrag im Zusammenhang

1 Von 2010 bis 2020 betrug das durchschnittliche Aufkommen im Monat November in Rheinland-Pfalz -5,9 Mio. Euro. 
Abbildung 1

Aufkommen der Körperschaftsteuer (vor Zerlegung) in Rheinland-Pfalz seit 2010

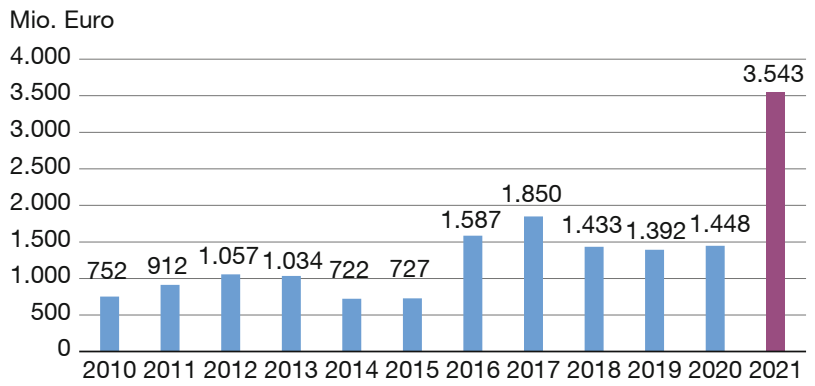

Quelle: Statistisches Bundesamt, BMF (2022b), eigene Darstellung.

mit der Geschäftsentwicklung des Mainzer Impfstoffherstellers steht, allerdings sind die genauen Hintergründe nicht bekannt, sodass im Weiteren darauf verzichtet wird, auch diese Aufkommensentwicklung dem BiontechFall zuzurechnen. Aber schon allein die extreme Steigerung des Körperschaftsteueraufkommens bewirkt, dass Rheinland-Pfalz erstmals als finanzstarkes Land im bundesstaatlichen Finanzausgleich erscheint. Ohne die Körperschaftsteuereinnahme von 1,7 Mrd. Euro läge die Finanzkraft unter dem Durchschnitt und Rheinland-Pfalz hätte Zuschläge im Finanzkraftausgleich (FKA) erhalten.

Allerdings sind damit die Wirkungen des Falls noch lange nicht vollständig erfasst. Zum einen ist dieser Steuerfall nicht auf die Körperschaftsteuer und das Jahr 2021 beschränkt, zum anderen sind zusätzlich die Auswirkungen auf weiteren Stufen des bundesstaatlichen Finanzausgleichs zu berücksichtigen. Deshalb werden im Folgenden die durch den Biontech-Fall ausgelösten Finanzströme und die Folgewirkungen für das bundesstaatliche Finanzgefüge genauer untersucht. Anzumerken ist, dass im Fall einer Berücksichtigung auch der erhöhten Einnahmen aus nichtveranlagten Steuern vom Ertrag die finanziellen Folgen des Biontech-Falles entsprechend größer ausfallen würden.

\section{Finanzströme des Biontech-Falles}

Das Jahr 2021 wurde wesentlich von den Auswirkungen der außergewöhnlich hohen Körperschaftsteuerzahlung im November bestimmt. $50 \%$ des originären Aufkommens verbleiben davon vorerst beim Land RheinlandPfalz und werden im Rahmen des FKA in der Finanzkraft von Rheinland-Pfalz berücksichtigt. Die Zerlegung dieser Körperschaftsteuereinnahmen wird mit einer Verzögerung von einem Quartal erst 2022 erfolgen.

Zusätzlich zur Körperschaftsteuer (KSt) wird auch die weitere Ertragsteuer der Unternehmen, die Gewerbesteuer (GewSt) fällig. Hierzu liegen jedoch noch keine belast- baren Statistiken vor, sodass die entsprechenden Zahlen bislang nur auf der Grundlage verschiedener Presseartikel abgeschätzt werden können (vgl. Tabelle 1).

Nach den vorliegenden Informationen wurden $2021 \mathrm{Ge}$ werbesteuerzahlungen in insgesamt ähnlicher Höhe geleistet wie bei der Körperschaftsteuer. Der Löwenanteil entfällt auf die Stadt Mainz, wo Biontech seinen Unternehmenssitz hat. Daneben unterhält das Unternehmen Betriebstätten in Idar-Oberstein (ebenfalls Rheinland-Pfalz) sowie in Marburg (Hessen) (inrlp.de, 2021; Hessenschau, 2021). Da die Gewerbesteuerhebesätze der drei Städte für das Jahr 2021 bekannt sind, kann der Gewerbesteuermessbetrag, die relevante Größe für weitere Verteilungsschritte, berechnet werden (vgl. Tabelle 2): Diese Gewerbesteuerzahlungen bzw. die Gewerbesteuermessbeträge haben Auswirkungen auf verschiedene Finanzausgleichssysteme, die teilweise mit erheblicher zeitlicher Verzögerung eintreten. Hier kann nur der bundesgesetzlich geregelte Teil dieser Auswirkungen beleuchtet werden.

Die dargestellten Körperschaftsteuerzahlungen werden auf die Betriebsstättenländer zerlegt. Dabei wird durch Verweis im Zerlegungsgesetz (ZerlG) auf § 29 GewStG im Regelfall derselbe Verteilungsschlüssel wie bei der Verteilung der Gewerbesteuer(messbeträge) angewandt. ${ }^{2}$ Entsprechend der Anteile bei den Gewerbesteuermessbeträgen wird hier von einem Betrag von 200 Mio. Euro ausgegangen, der im Rahmen der Körperschaftsteuerzerlegung an das Land Hessen wegen der Betriebsstätte von Biontech in Marburg fließt. Diese Zerlegung erfolgt quartalsweise nachlaufend, d.h. ein Teil der Körperschaftsteuereinnahmen, die im IV. Quartal 2021 in Rheinland-Pfalz eingegangen sind, wird erst im I. Quartal 2022 an das Land Hessen abgegeben.

Von den Gewerbesteuereinnahmen müssen die Gemeinden Umlagen einerseits an den Bund und das jeweilige Land nach bundesweit geltenden Regeln und andererseits nach landesinternen Regeln z.B. an die Landkreise abführen. In diesem Beitrag werden ausschließlich die nach Bundesgesetz geregelten Umlagen betrachtet (vgl. Tabelle 3). ${ }^{3}$ Die Abrechnung des IV. Quartals erfolgt im Regelfall im Januar des Folgejahrs.

\section{Finanzausgleichswirkungen des Biontech-Falles 2021}

Selbst wenn man den außergewöhnlichen Steuerfall von Biontech als reinen Einmaleffekt ansieht, wird es über mehrere Jahre hinweg zu Folgewirkungen im System des bundesstaatlichen Finanzausgleichs kommen. Dabei schlägt sich in der aktuellen Jahresabrechnung des

$2 \S 2$ Abs. 1 ZerlG.

$3 \S 6$ Gemeindefinanzreformgesetz. 
Tabelle 1

Annahmen: Zusätzliche Steuereinnahmen 2021

in Mio. Euro

\begin{tabular}{lrrr} 
Körperschaftsteuer & \multicolumn{3}{c}{ Gewerbesteuer } \\
\hline Finanzamt in Rheinland-Pfalz & 1.700 & Gemeindekassen der Städte & 1.670 \\
\hline davon & \multicolumn{2}{c}{ davon } \\
\hline Bund & 850 & Mainz & 1100 \\
\hline Land & 850 & Idar-Oberstein & 200 \\
\hline & & Marburg & 370 \\
\hline
\end{tabular}

Quelle: BMF (2022b), inrlp.de (2021), Hessenschau (2021), eigene Berechnungen.

Tabelle 2

Rückrechnung Gewerbesteuer-Messbeträge 2021

\begin{tabular}{lccccc} 
& $\begin{array}{c}\text { Aufkommen } \\
\text { (in Mio. Euro) }\end{array}$ & $\begin{array}{c}\text { Hebesatz } \\
\text { (in \%) }\end{array}$ & $\begin{array}{c}\text { Messbetrag } \\
\text { (in Mio. Euro) }\end{array}$ \\
\hline Mainz & 1.100 & $/$ & 440 & $=$ & 250 \\
\hline Idar-Oberstein & 200 & $/$ & 420 & $=$ & 48 \\
\hline Marburg & 370 & $/$ & 400 & $=$ & 93 \\
\hline Summe & 1.670 & $/$ & & & 390
\end{tabular}

Quelle: inrlp.de (2021), Hessenschau (2021), DIHK (2021), eigene Berechnungen.

Tabelle 3

Annahmen: Gewerbesteuerumlagen (GU) Bund/Land für die Gewerbesteuer 2021

\begin{tabular}{ccc}
$\begin{array}{c}\text { Messbetrag } \\
\text { (in Mio. Euro) }\end{array}$ & $\begin{array}{c}\text { Vervielfältiger } \\
\text { (in \%) }\end{array}$ & Umlage \\
(in Mio. Euro) \\
\hline
\end{tabular}

\begin{tabular}{lccccc}
\hline Gewerbesteuer- & & & & & \\
Umlage Bund & 390 & x & 14,5 & $=$ & 57 \\
\hline $\begin{array}{l}\text { Gewerbesteuer- } \\
\text { Umlage Land RP }\end{array}$ & 298 & x & 20,5 & $=$ & 61 \\
\hline $\begin{array}{l}\text { Gewerbesteuer- } \\
\text { Umlage Land HE }\end{array}$ & 93 & x & 20,5 & $=$ & 19 \\
\hline Summe & & & & & 137 \\
\hline
\end{tabular}

Quelle: eigene Berechnungen auf Basis zuvor beschriebener Annahmen.

Finanzausgleichs 2021 ausschließlich die Körperschaftsteuerzahlung im November in Rheinland-Pfalz nieder. Das Jahr 2022 wird hingegen maßgeblich von den Auswirkungen der Gewerbesteuerzahlungen 2021 auf die Gemeindefinanzkraft 2022 geprägt.

\section{Das Ausgleichsjahr 2021}

Die zusätzlichen Körperschaftsteuereinnahmen von 850 Mio. Euro führen rechnerisch zu einer Erhöhung der relativen Finanzkraft von Rheinland-Pfalz um 3,5 Prozentpunkte. Damit erreicht Rheinland-Pfalz erstmals eine überdurchschnittliche Finanzkraft und wird im FKA mit Abschlägen von 287 Mio. Euro ein finanzstarkes Land. Ohne diese Körperschaftsteuereinnahmen von Biontech hätte Rheinland-
Pfalz Zuschläge von 164 Mio. Euro erhalten. Insgesamt führen die Mehreinnahmen bei der Körperschaftsteuer zu einer Verschlechterung im FKA von 451 Mio. Euro und folglich zu einer Abschöpfungsquote von $53 \%$.

Gleichzeitig entfallen bei Rheinland-Pfalz auch die finanzkraftabhängigen allgemeinen Bundesergänzungszuweisungen (BEZ), was einen zusätzlichen Verlust von 36 Mio. Euro bedeutet. Darüber hinaus ist die Gewährung der 2020 neu eingeführten „Forschungs-BEZ“ nach § 11 Abs. 6 Finanzausgleichsgesetz (FAG) ausdrücklich auf „leistungsschwache Länder" beschränkt. Als nunmehr finanzstarkes Land kommt Rheinland-Pfalz nicht mehr als Empfänger infrage, womit das Land weitere Zuweisungen in Höhe von 77 Mio. Euro verliert.

Von dem originären Körperschaftsteuermehraufkommen in Höhe von 850 Mio. Euro verbleiben nach der Jahresabrechnung für 2021 somit lediglich 286 Mio. Euro in Rheinland-Pfalz. Wegen der zusätzlichen Verluste bei den BEZ steigt die Abschöpfungsquote auf über $66 \%$. Dabei macht sich die sogenannte Prämienregelung nach $\S 7$ Abs. 3 FAG deutlich bemerkbar, wonach Rheinland-Pfalz $12 \%$ der überdurchschnittlichen Mehreinnahmen nicht bei der Finanzkraft angerechnet werden. Ohne die Prämienregelung läge die Abschöpfungsquote sogar bei $72 \% .500$ Mio. Euro fließen an die anderen Länder und auch der Bund wird wegen der im Saldo sinkenden BEZ zusätzlich zu den originären Steuermehreinnahmen um 34 Mio. Euro entlastet.

\section{Das Ausgleichsjahr 2022}

Im Ausgleichsjahr 2022 werden sich die Zahlungsströme, die Körperschaftsteuerzerlegung sowie die Abführung der Gewerbesteuerumlagen unmittelbar auswirken. Die finanziellen Folgen für Rheinland-Pfalz sind jetzt davon abhängig, in welcher finanziellen Ausgangsposition sich das Land 2022 befindet, ob es weiter ein finanzstarkes Land bleibt oder wieder finanzschwach wird. Unter der (extrem restriktiven) Annahme, dass der Biontech-Steuerfall ein reiner Einmaleffekt bleibt, also 2022 keine weiteren Körperschaftbzw. Gewerbesteuerzahlungen erfolgen, würde sich auf Basis der Finanzkraftverhältnisse 2021 für Rheinland-Pfalz eine relative Finanzkraft von 98,9\% ergeben. ${ }^{4}$ Damit wäre das Land wieder Empfängerland im FKA und hätte Anspruch auf allgemeine BEZ sowie auf Forschungs-BEZ.

Durch die Zerlegung der Körperschaftsteuer und durch den Zufluss des Länderanteils an der Gewerbesteuerum-

4 Und bei Nichtberücksichtigung der Prämienregel nach $\S 7$ Abs. 3 FAG. Wird auch der Einmaleffekt bei den nichtveranlagten Steuern vom Ertrag zusätzlich berücksichtigt, sinkt die Finanzkraft von Rheinland-Pfalz sogar auf $95,8 \%$. 
Tabelle 4

Auswirkung der Körperschaftsteuermehreinnahmen im Ausgleichsjahr 2021

in Mio. Euro

\begin{tabular}{lcccc} 
& $\begin{array}{c}\text { Rheinland- } \\
\text { Pfalz }\end{array}$ & Hessen & $\begin{array}{c}\text { übrige } \\
\text { Länder }\end{array}$ & Bund \\
\hline KSt-Mehreinnahmen & 850 & & & 850 \\
\hline Veränderung Finanzkraft & $3,5 \%$ & $-0,2 \%$ & & \\
\hline FKA & -451 & 30 & 421 & - \\
\hline allgemeine BEZ & -36 & 0 & 79 & -43 \\
\hline übrige BEZ & -77 & 0 & 0 & 77 \\
\hline Finanzausgleich insgesamt & -564 & 30 & 500 & 34 \\
\hline $\begin{array}{l}\text { Steuermehreinnahmen } \\
\text { nach Finanzausgleich }\end{array}$ & 286 & 30 & 500 & 884 \\
\hline
\end{tabular}

Quelle: eigene Berechnungen auf Basis zuvor beschriebener Annahmen.

lage sinkt im Saldo die Steuerkraft des Landes. Darüber hinaus sinkt auch die Steuerkraft der rheinland-pfälzischen Kommunen wegen der abfließenden Gewerbesteuerumlagen (Landes- und Bundesanteil). Insgesamt geht die relative Finanzkraft von Rheinland-Pfalz um weitere 1,1 Prozentpunkte zurück. Bei Hessen kommt es dagegen zu einer Finanzkraftzunahme um +0,6 Prozentpunkte. Wie die Tabelle 5 zeigt, werden die originären Verluste des Landeshaushalts von Rheinland-Pfalz durch den FKA und die finanzkraftabhängigen allgemeinen BEZ wegen der Anrechnung der gesunkenen Gemeindefinanzkraft sogar überkompensiert. Die ursprünglichen Steuermindereinnahmen von 139 Mio. Euro verwandeln sich in ein Plus von 61 Mio. Euro.

Durch die hohe Biontech-Steuerzahlung, insbesondere an die Stadt Mainz, steigt jedoch die Gewerbesteuerkraft von Rheinland-Pfalz massiv an, was enorme Konsequenzen für den FKA 2022 hat, da nach § 8 Abs. 2 FAG die „im Ausgleichsjahr aufgekommenen einzelnen Realsteuern im Verhältnis der länderweisen Grundbeträge dieser Steuern in dem dem Ausgleichsjahr vorausgehenden Kalenderjahr verteilt werden“.

Die Gewerbesteuerzahlungen von Biontech 2021 an Mainz, Idar-Oberstein und Marburg entsprechen rechnerisch einem Zuwachs an Grundbeträgen der Gewerbesteuer von rund 300 Mio. Euro in Rheinland-Pfalz und von etwas mehr als 90 Mio. Euro in Hessen. Auf der Basis der Gewerbesteuerkraft 2020 würde dies einen Zuwachs von fast $60 \%$ für Rheinland-Pfalz bedeuten. Damit steigt der Anteil des Landes Rheinland-Pfalz am bundesweiten Gewerbesteueraufkommen um 2,4 Prozentpunkte, während sich alle anderen Länder (mit Ausnahme von Hessen wegen der Betriebsstätte Marburg) entsprechend verschlechtern, wie Abbildung 2 zeigt. Basierend auf dem bei der Abrechnung des Ausgleichsjahres 2021 berücksichtigten
Tabelle 5

Auswirkung der Körperschaftsteuerzerlegung und Gewerbesteuerumlagen im Ausgleichsjahr 2022

in Mio. Euro

\begin{tabular}{lrrrrr} 
& $\begin{array}{c}\text { Rhein- } \\
\text { land-Pfalz }\end{array}$ & \multicolumn{2}{c}{ übrige } \\
& -200 & 200 & & \\
\hline KSt-Zerlegung & 61 & 19 & 57 \\
\hline Gewerbesteuerumlage & -139 & 219 & & 57 \\
\hline Auswirkung vor Finanzausgleich & & & & \\
\hline Veränderung Gemeindefinanzkraft & -104 & -32 & & \\
(100\%) wegen Gewerbesteuerumlage & $-1,1 \%$ & $0,6 \%$ & & \\
\hline Veränderung Finanzkraft & 136 & -124 & -12 & - \\
\hline FKA & 64 & 0 & -2 & -62 \\
\hline allgemeine BEZ & 0 & 0 & -9 & 9 \\
\hline übrige BEZ & 200 & -124 & -24 & -52 \\
\hline Finanzausgleich insgesamt & 61 & 95 & -24 & 4 \\
\hline Wirkung nach Finanzausgleich & & & &
\end{tabular}

Quelle: eigene Berechnungen auf Basis zuvor beschriebener Annahmen.

Gewerbesteueraufkommen von 55,8 Mrd. Euro bedeutet der Zuwachs der Gewerbesteuerkraft um 2,4 Prozentpunkte, dass Rheinland-Pfalz 2022 1,2 Mrd. Euro mehr Gewerbesteuer angerechnet wird als im Vorjahr, wovon $75 \%$ als zusätzliche Finanzkraft im FKA berücksichtigt werden. Isoliert betrachtet, ergibt sich allein durch die veränderte Gewerbesteuerkraft ein Finanzkraftzuwachs von rund 5 Prozentpunkten. Damit wird Rheinland-Pfalz voraussichtlich auch 2022 ein finanzstarkes Land mit den entsprechenden Konsequenzen beim FKA und bei den BEZ. ${ }^{5}$

Wie Tabelle 6 zeigt, führt die 2021 gestiegene Gewerbesteuerkraft für Rheinland-Pfalz zu einer Verschlechterung im FKA von 629 Mio. Euro. Dazu kommen Verluste bei den allgemeinen BEZ von 90 Mio. Euro sowie der (nochmalige) Verlust der Forschungs-BEZ, sodass sich die Verluste im Finanzausgleich auf insgesamt 796 Mio. Euro summieren. Auch in Hessen kommt es wegen der gestiegenen Gemeindefinanzkraft zu Verlusten im Finanzausgleich, während alle anderen Länder um fast 1 Mrd. Euro entlastet werden.

\section{Gesamteffekt}

Insgesamt hat also der Biontech-Fall selbst dann 2022 erhebliche finanzielle Auswirkungen, wenn man die unrealistische Annahme trifft, dass 2022 keine weiteren Ertragssteuern vom Impfstoffhersteller abgeführt werden.

5 Bereinigt man die Berechnungsbasis zusätzlich um den Einmaleffekt bei den nichtveranlagten Steuern vom Ertrag ergibt sich zwar eine unterdurchschnittliche Finanzkraft für Rheinland-Pfalz, diese bleibt aber mit $99,7 \%$ so nahe an der durchschnittlichen Finanzkraft, dass das Land weiterhin nicht leistungsschwach wird und damit keine allgemeinen BEZ und keine Forschungs-BEZ beziehen kann. 


\section{Abbildung 2}

Veränderung der Anteile an den Grundbeträgen der Gewerbesteuer durch den Biontech-Fall

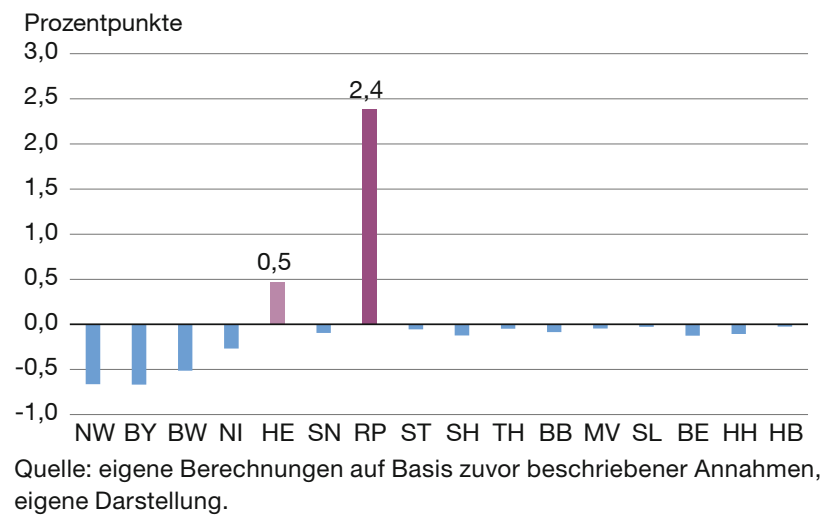

Insgesamt kommt es zu den in Tabelle 7 dargestellten Zahlungsflüssen und Auswirkungen im Finanzausgleich 2021 und 2022: Hier zeigt sich, dass von der ursprünglichen Steuersumme von 3,37 Mrd. Euro, die im IV. Quartal 2021 von Biontech vorwiegend in Mainz abgeführt wurde, nach Durchführung der Steuerverteilung und des Finanzausgleichs nur knapp 750 Mio. Euro in Rheinland-Pfalz verbleiben. Es fließen nicht nur der Bundesanteil und die Zerlegungsanteile $a b$, sondern es kommt auch über die beiden Jahre zu enormen Finanzausgleichseffekten, die sich für das Land Rheinland-Pfalz auf insgesamt rund 1,15 Mrd. Euro belaufen. Damit kommt es zu einer paradoxen Situation: In Rheinland-Pfalz profitieren allein zwei Kommunen in einem enormen Umfang, da die Gewerbesteuerzahlungen weitgehend in den Kommunalkassen verbleiben (unter Ausblendung eventueller landesrechtlicher Regelungen). Der Landeshaushalt weist dagegen trotz der hohen originären Körperschaftsteuermehreinnahmen unter Berücksichtigung aller Folgewirkungen ein Netto-Defizit von 450 Mio. Euro aus. Hierfür ist insbesondere die gestiegene Gemeindesteuerkraft verantwortlich, die zu zusätzlichen Verlusten des Landes im FKA und bei den BEZ führt. Drastisch ausgedrückt führt der BiontechFall dazu, dass die Stadt Mainz 2022 schuldenfrei sein wird, wie in verschiedenen Pressemeldungen zu lesen war, das Land Rheinland-Pfalz und damit - über den Automatismus des regelgebundenen kommunalen Finanzausgleichs - auch alle anderen Kommunen in RheinlandPfalz eventuell sogar weniger Geld zur Verfügung haben werden. ${ }^{6}$

6 Selbst wenn man den Einmaleffekt der nichtveranlagten Steuern noch zum Biontech-Fall hinzurechnen würde, verbleibt für den Landeshaushalt insgesamt ein Nettoverlust, da der Sondereffekt der nichtveranlagten Steuern vom Ertrag im Ausgleichsjahr für Rheinland-Pfalz allenfalls Nettomehreinnahmen nach Finanzausgleich von 305 Mio. Euro gebracht hat.
Tabelle 6

Auswirkung der veränderten Gewerbesteuerkraft im Ausgleichsjahr 2022

in Mio. Euro

\begin{tabular}{lcccc} 
& $\begin{array}{c}\text { Rheinland- } \\
\text { Pfalz }\end{array}$ & Hessen Länder & Bund \\
\hline $\begin{array}{l}\text { zusätzliche Gewerbe- } \\
\text { steuergrundbeträge 2021 }\end{array}$ & 298 & 93 & & - \\
\hline $\begin{array}{l}\text { im FA } 2022 \text { zusätzlich berück- } \\
\text { sichtigte Gewerbesteuer (100 \%) }\end{array}$ & 1.331 & 262 & -1.594 & - \\
\hline Veränderung Finanzkraft & $4,9 \%$ & $0,6 \%$ & & \\
\hline FKA & -629 & -124 & 753 & - \\
\hline allgemeine BEZ & -90 & 0 & 112 & -21 \\
\hline übrige BEZ & -77 & 0 & 108 & -30 \\
\hline Finanzausgleich insgesamt & -797 & -124 & 972 & -52 \\
\hline
\end{tabular}

Quelle: eigene Berechnungen auf Basis zuvor beschriebener Annahmen.

Ähnlich, wenn auch lange nicht so drastisch, ist die Situation in Hessen, wo über den Produktionsstandort Marburg ebenfalls hohe Gewerbesteuereinnahmen zu verzeichnen sind. Weiterhin stehen dem Land Anteile der Körperschaftsteuer zu, die über die Zerlegung 2022 kassenwirksam werden, sodass der Biontech-Fall zunächst knapp 600 Mio. Euro an Steuereinnahmen in die hessischen Finanzkassen spült. Jedoch werden auch diese Einnahmen durch den bundesstaatlichen Finanzausgleich gemindert, sodass letztlich lediglich rund 340 Mio. Euro im Land Hessen verbleiben. Auch hier ist festzuhalten, dass das Steuerplus nahezu vollständig auf der kommunalen Ebene verzeichnet wird.

Weitere Profiteure dieses außergewöhnlichen Steuerfalls sind die anderen Länder und der Bund. Der starke Anstieg der rheinland-pfälzischen Finanzkraft und der damit verbundene Wechsel vom Nehmer- zum Geberland führt dazu, dass die Finanzkraftzuschläge der finanzschwachen Länder zunehmen und die Abschläge der anderen finanzstarken Länder sinken. Der Bund profitiert vor allem von seinem Anteil an der Körperschaftsteuer, aber auch von der Gewerbesteuerumlage, während sich im Saldo bei den $B E Z$ eine Belastung ergibt.

\section{Szenario-Analyse mit Steuermehreinnahmen 2022}

Damit stellt sich die Frage nach den langfristigen Folgen von Biontech: Wie ausgeführt, hat der Steuerfall von Biontech 2021 so weitreichende Auswirkungen, dass das bisher stets finanzschwache Land Rheinland-Pfalz wahrscheinlich für zwei Jahre ein finanzstarkes Land wird. Außerdem ist kaum damit zu rechnen, dass es bei einer nur einmaligen Steuerabführung des Impfstoffherstellers bleibt. Angesichts der Entwicklung der weltweiten Coronapandemie ist 
Tabelle 7

Gesamtwirkung des Biontech-Falles (als Einzelfall) in Mio. Euro

Rheinland-Pfalz Hessen

Kom- Kom- übrige

Land munen Landmunen Länder Bund

\begin{tabular}{lrrrrrr}
\hline 2021 & & & & & & \\
\hline KSt-Mehreinnahmen & 850 & & & & & 850 \\
\hline - Auswirkungen FA 2021 & -564 & & 30 & & 500 & 34 \\
\hline Gewerbesteuer & & 1.300 & & 370 & & \\
\hline Insgesamt 2021 & 286 & 1.300 & 30 & 370 & 500 & 884 \\
\hline 2022 & & & & & & \\
\hline KSt-Zerlegung & -200 & & 200 & & & \\
\hline Gewerbesteuerumlage & 61 & -104 & 19 & -32 & & 57 \\
\hline
\end{tabular}

Veränderung Grundbetrag (298) (93)

GewSt 2021

\begin{tabular}{lrrrrrr}
\hline - Auswirkungen FA 2022 & -596 & & -248 & & 948 & -104 \\
\hline Insgesamt 2022 & -735 & -104 & -29 & -32 & 948 & -47 \\
\hline Insgesamt & -450 & 1.196 & 1 & 338 & 1.448 & 837 \\
\hline
\end{tabular}

Quelle: eigene Berechnungen auf Basis zuvor beschriebener Annahmen.

davon auszugehen, dass die Nachfrage nach Corona-Impfstoffen weiter hoch bleiben wird und Biontech auch weiterhin Gewinne ausweisen - und versteuern - dürfte.

Über die künftig zu erwartenden Steuerabführungen kann nur spekuliert werden. Dennoch ist davon auszugehen, dass zumindest 2022 noch erhebliche Steuerzahlungen von Biontech zu erwarten sind. Da abzusehen ist, dass Rheinland-Pfalz auch 2022 ein finanzstarkes Land bleibt, werden zusätzliche Steuereinnahmen lediglich durch den FKA gemindert. Die zusätzlichen Effekte über die BEZ, die 2021 noch eine bedeutende Rolle spielen, entfallen. Somit könnte Rheinland-Pfalz damit rechnen, dass weitere Körperschaftsteuerzahlungen von Biontech nur noch zu etwa $60 \%$ abgeschöpft werden.

Um die Folgen für das System des bundesstaatlichen Finanzausgleichs 2022 abschätzen zu können, wird hier in einer Beispielrechnung unterstellt, dass 2022 etwa die Hälfte des den Steuerzahlungen 2021 zugrundeliegenden Gewinns anfällt und versteuert wird. Dies würde für Rheinland-Pfalz 2022 einen weiteren Zufluss an Körperschaftsteuer von 850 Mio. Euro bedeuten, wovon nach Abführung des Bundesanteils und der Zerlegungsanteile Hessens knapp 330 Mio. Euro in der Landeskasse verbleiben würden. Etwas komplizierter ist die Abschätzung der zu erwartenden Mehreinnahmen bei der Gewerbesteuer, da nach den vorliegenden Informationen zumindest bei zwei beteiligten Städten deutliche Herabsetzungen der Hebesätze zu erwarten sind. So hat die Stadt Mainz bereits die
Senkung des Hebesatzes für die Gewerbesteuer von 440 auf 310 beschlossen (SWR, 2021). Auch Marburg plant nach den vorliegenden Informationen eine Senkung auf $357 \%$ (Mittelhessen, 2021). Lediglich Idar-Oberstein hat für 2022 den Hebesatz unverändert gelassen (Stadtrat Idar-Oberstein, 2021). ${ }^{7}$ Geht man von diesen Hebesätzen für 2022 aus und unterstellt, dass sich das Verhältnis von Körperschaftsteuerzahlung und Gewerbesteuermessbetrag gegenüber 2021 nicht ändert (d. h. die Gewerbesteuermessbeträge der drei beteiligten Städte halbieren sich gegenüber dem Jahr 2021), ergeben sich die in Tabelle 8 dargelegten Schätzungen für das Gewerbesteueraufkommen und die Gewerbesteuerumlagen 2022.

Unter diesen Annahmen belaufen sich die zusätzlichen Gewerbesteuereinnahmen 2022 auf rund 650 Mio. Euro, wovon 490 Mio. Euro in Rheinland-Pfalz und 160 Mio. Euro in Hessen aufkommen. Berücksichtigt man darüber hinaus die Körperschaftsteuerzahlungen, die Zerlegung zwischen Rheinland-Pfalz und Hessen sowie die Abführungen der Gewerbesteuerumlagen, ergeben sich die in Tabelle 9 dargelegten originären Steuermehreinnahmen der verschiedenen staatlichen Ebenen 2022.

Für den Finanzausgleich des Ausgleichsjahrs 2022 sind dabei allerdings nur die Nettosteuerzuflüsse an die Länder Rheinland-Pfalz und Hessen von Bedeutung. ${ }^{8}$ Die Veränderung der Gewerbesteuerkraft wird wieder erst mit einer Verzögerung von einem Jahr im Finanzausgleich 2023 relevant. Die Körperschaftsteuereinnahme (unter Berücksichtigung der Zerlegung) und der Zufluss an Gewerbesteuerumlage führen zu einer weiteren deutlichen Erhöhung der relativen Finanzkraft von Rheinland-Pfalz um 1,5 Prozentpunkte, wodurch in der Modellrechnung für das Jahr 2022 mit 104,2 \% eine Finanzkraft erreicht wird, die deutlich über dem Durchschnitt liegt. Auch die hessische Finanzkraft steigt aufgrund der Zuflüsse im Rahmen der Körperschaftsteuerzerlegung und der Gewerbesteuerumlage, wenn auch lange nicht so stark wie im Nachbarland.

Da Rheinland-Pfalz bereits durch die Berücksichtigung der Realsteuerkraft 2021 zum finanzstarken Land wird, hat die weitere Steigerung der Finanzkraft jetzt nur noch Auswirkungen im FKA und es gibt keine weiteren Effekte bei den BEZ. Wie Tabelle 10 zeigt, steigen die Abschläge von Rheinland-Pfalz im Rahmen des FKA um 188 Mio. Euro, sodass dem Landeshaushalt Mehreinnahmen von

7 Vgl. Beschluss des Stadtrats von Idar-Oberstein in seiner Sitzung am 15. Dezember 2021 für den Stadthaushalt für 2022. In der mitbeschlossenen Haushaltssatzung wurde auch der GewSt-Hebesatz für 2022 gleichbleibend mit $420 \%$ beschlossen.

8 Die möglichen Phasenverschiebungen um ein Quartal bei der Zerlegung und bei den Gewerbesteuerumlagen werden aus Vereinfachungsgründen hier nicht berücksichtigt. 
Tabelle 8

Geschätzte Gewerbesteuer und

Gewerbesteuerumlagen für 2022

in Mio. Euro

\begin{tabular}{lrrrrr} 
& $\begin{array}{c}\text { Messbetrag } \\
\text { GewSt }\end{array}$ & $\begin{array}{c}\text { Hebesatz } \\
\text { (neu) }\end{array}$ & $\begin{array}{r}\text { Gewerbe- } \\
\text { steuer }\end{array}$ & $\begin{array}{r}\text { GU } \\
\text { Land }\end{array}$ & $\begin{array}{c}\text { GU } \\
\text { Bund }\end{array}$ \\
\hline Mainz & 125 & $310 \%$ & 388 & 26 & 18 \\
\hline Idar-Oberstein & 24 & $420 \%$ & 100 & 5 & 3 \\
\hline Rheinland-Pfalz & & & 488 & 31 & \\
\hline Marburg (Hessen) & 46 & $357 \%$ & 165 & 9 & 7 \\
\hline Summe & 195 & & 653 & 40 & 28 \\
\hline
\end{tabular}

Quelle: SWR (2021), Mittelhessen (2021), Stadtrat Idar-Oberstein (2021), eigene Berechnungen auf Basis zuvor beschriebener Annahmen.

169 Mio. Euro verbleiben. Diese Netto-Mehreinnahmen genügen aber nicht, die in Tabelle 7 abgeleiteten Verluste des Landeshaushalts aus dem Biontech-Fall 2021 auszugleichen. Selbst unter optimistischen Annahmen über die weitere Gewinnentwicklung von Biontech wird der Landeshaushalt in Rheinland-Pfalz kurzfristig hier nicht mit einer Entlastung rechnen können.

Der überwiegende Teil der zu erwartenden (Körperschaft-)Steuermehreinnahmen von Biontech 2022 kommt mit 267 Mio. Euro den übrigen Ländern zugute, da die Zu- bzw. Abschläge im FKA entsprechend steigen bzw. sinken und die allgemeinen BEZ wegen der vergrößerten Finanzkraftdisparitäten zunehmen.

\section{Bleibt Rheinland-Pfalz dauerhaft finanzstark?}

Nach der bisherigen Analyse erscheint es durchaus wahrscheinlich, dass die enormen Sondereffekte im IV. Quartal 2021 schon dazu führen, dass Rheinland-Pfalz auch im nächsten Jahr finanzstark sein wird. Darüber hinaus dürfte auch weiterhin mit einem positiven Effekt von Biontech auf das rheinland-pfälzische Steueraufkommen zu rechnen sein. Hier drängt sich die Frage auf, wie hoch das zusätzliche Steueraufkommen ausfallen müsste, damit sich Rheinland-Pfalz auf Dauer als finanzstarkes Land etablieren kann. Zur Beantwortung dieser Frage wird im Folgenden ein stark vereinfachtes Szenario entwickelt. Ausgehend von den jetzigen Aufkommensrelationen zwischen Körperschaft- und Gewerbesteuer, (bzw. exakter Gewerbesteuermessbeträgen) und unter der Annahme konstanter Hebe- und Umlagesätze wird in einer Zielwertanalyse das Körperschaftsteueraufkommen der Firma Biontech ermittelt, mit dem Rheinland-Pfalz eine Finanzkraft von exakt $100 \%$ erreicht. Hierbei wird das exorbitante Aufkommen der nichtveranlagten Steuern vom Ertrag im Dezember 2021 in Rheinland-Pfalz aus der Basis herausgerechnet, da mit diesem Aufkommen kaum auf Dauer gerechnet werden kann.
Tabelle 9

Annahmen über zusätzliche Steuereinnahmen 2022 in Mio. Euro

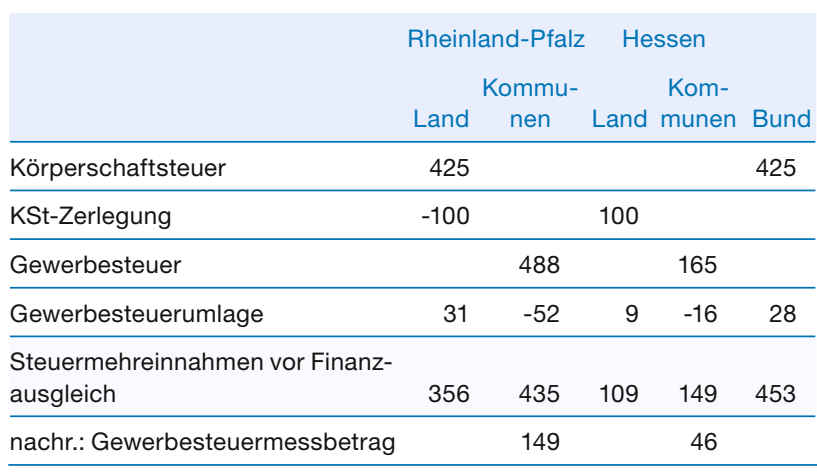

Quelle: eigene Berechnungen auf Basis zuvor beschriebener Annahmen.

Dabei zeigt sich, dass für dieses Ziel zusätzliche Steuereinnahmen bei etwa der Hälfte der im IV. Quartal 2021 verzeichneten Mehreinnahmen des Biontech-Falles nötig wären. In der Szenario-Analyse wird eine Finanzkraft von exakt $100 \%$ für Rheinland-Pfalz dann erreicht, wenn eine KSt-Zahlung von insgesamt 895 Mio. Euro erfolgt, die bei konstanten Verteilungsverhältnissen auch Gewerbesteuerabführungen von 688 Mio. Euro mit sich bringt. Die genaue Verteilung der Steuermehreinnahmen sowie die daraus folgenden Umlagen sind der Tabelle 11 zu entnehmen.

Hier zeigt sich ein paradoxes Ergebnis: Unter den getroffenen Annahmen kommt es langfristig zu so großen negativen Finanzausgleichseffekten in Rheinland-Pfalz, dass von den ursprünglichen Mehreinnahmen der Landes- und Kommunalebene in Höhe von 832 Mio. Euro kaum etwas im Land verbleibt. Die Verluste des Landeshaushalts nach Finanzausgleich in Höhe von 446 Mio. Euro kompensieren fast vollständig die Gewinne der Kommunen mit 459 Mio. Euro. Dies liegt unter anderem daran, dass in diesem Szenario negative Abschöpfungseffekte kumulativ auftreten:

- Der direkte Finanzkraftausgleichseffekt zusätzlicher eigener Einnahmen (KSt),

- der Finanzkraftausgleichseffekt über die gestiegene Gewerbesteuerkraft,

- beide verstärkt über die Wirkung der sinkenden bzw. wegfallenden BEZ.

Ein entscheidender Punkt für die extrem hohe Abschöpfungsquote von $98,5 \%$ liegt aber darin, dass bei der Zurechnung der Gewerbesteuer zur Finanzkraft des Landes Rheinland-Pfalz nicht die tatsächlichen Gewerbesteuermehreinnahmen von 514 Mio. Euro berücksichtigt werden, sondern die Gewerbesteuerkraft. Vereinfacht ausgedrückt erhält man diese, wenn das Gewerbesteueraufkommen durch den jeweiligen tatsächlichen Hebesatz dividiert und 
Tabelle 10

Auswirkungen unterstellter Steuermehreinnahmen im Finanzausgleich 2022

in Mio. Euro

\begin{tabular}{cl}
$\begin{array}{c}\text { Rhein- } \\
\text { land-Pfalz Hessen }\end{array}$ & Läbrige \\
\hline
\end{tabular}

Steuermehreinnahmen vor Finanz-

\begin{tabular}{llll} 
ausgleich & 356 & 109 & 453 \\
\hline
\end{tabular}

Veränderung Gemeindefinanzkraft

\begin{tabular}{lrrrr} 
(100\%) wegen GU & -52 & -16 & & \\
\hline Veränderung Finanzkraft & $1,5 \%$ & $0,2 \%$ & & \\
\hline FKA & -187 & -42 & 229 & - \\
\hline allgemeine BEZ & 0 & 0 & 43 & -43 \\
\hline übrige BEZ & 0 & 0 & -5 & 5 \\
\hline Wirkung Finanzausgleich insgesamt & -187 & -42 & 267 & -38 \\
\hline Wirkung nach Finanzausgleich & 169 & 67 & 267 & 415
\end{tabular}

Quelle: eigene Berechnungen auf Basis zuvor beschriebener Annahmen.

anschließend mit dem bundesdurchschnittlichen Hebesatz multipliziert wird. Die Normierung ist zur sachgerechten Ermittlung der kommunalen Finanzkraft zum Zweck des Finanzausgleichs und zur Vermeidung von Fehlanreizen unumgänglich. Ansonsten könnten durch Hebesatzsenkungen verursachte Aufkommensminderungen auf andere Länder abgewälzt werden (Scherf, 2000). Somit hängt die Veränderung der Gewerbesteuerkraft einer Gemeinde neben der Wachstumsrate des Gewerbesteueraufkommens auch maßgeblich vom Hebesatzverhältnis ab. Je stärker der individuelle Hebesatz vom durchschnittlichen Hebesatz nach unten abweicht, umso stärker ist der (kalkulatorische) Zuschlag der Gewerbesteuerkraft, wie sich am Beispiel der Stadt Mainz eindrucksvoll zeigt: Da die Stadt Mainz ab 2022 den Hebesatz der Gewerbesteuer massiv auf 310 und damit weit unter den Bundesdurchschnitt von 400 senkt, werden dem Land Rheinland-Pfalz in der Szenario-Rechnung Gewerbesteuereinnahmen von 712 Mio. Euro zugerechnet. Diese werden zu $75 \%$ im FKA berücksichtigt, sodass die Finanzkraft des Landes RheinlandPfalz um 534 Mio. Euro und damit stärker als der tatsächliche Zufluss an Gewerbesteuer steigt. Dies hat erhebliche zusätzliche Abschöpfungen im Finanzausgleich zur Folge. Dagegen kann Hessen mit der kleineren Betriebsstätte Marburg mit deutlich höheren verbleibenden Mehreinnahmen rechnen, da es als finanzstarkes Land nicht von den negativen Abschöpfungseffekten der BEZ betroffen ist. Erst wenn Rheinland-Pfalz noch höhere Steuermehreinnahmen generieren kann, würde es auch dauerhaft größere Nettogewinne erzielen können.

\section{Fazit und Ausblick}

Der Fall Biontech zeigt, dass der Wechsel vom finanzschwachen zum finanzstarken Land durchaus möglich ist.
Tabelle 11

\section{Szenario: Zusätzliches KSt-Aufkommen von} Biontech von 895 Mio. Euro

in Mio. Euro

\begin{tabular}{|c|c|c|c|c|c|c|}
\hline & Rheinla & and-Pfalz & $\mathrm{He}$ & ssen & & \\
\hline & Land & $\begin{array}{l}\text { Kom- } \\
\text { munen }\end{array}$ & Land & $\begin{array}{l}\text { Kom- } \\
\text { munen }\end{array}$ & $\begin{array}{l}\text { übrige } \\
\text { Länder }\end{array}$ & Bund \\
\hline KSt (nach Zerlegung) & 342 & & 106 & & & 448 \\
\hline Gewerbesteuer & & 514 & & 174 & & \\
\hline Gewerbesteuerumlage & 32 & -55 & 10 & -17 & & 30 \\
\hline $\begin{array}{l}\text { Mehreinnahme vor Finanz- } \\
\text { ausgleich }\end{array}$ & 374 & 459 & 116 & 157 & 0 & 477 \\
\hline Veränderung Finanzkraft & $4,2 \%$ & & $0,5 \%$ & & & \\
\hline FKA & -533 & & -111 & & 644 & - \\
\hline allgemeine BEZ & -210 & & 0 & & 105 & 104 \\
\hline übrige BEZ & -77 & & 0 & & 53 & 25 \\
\hline Finanzausgleich insgesamt & -820 & & -111 & & 802 & 129 \\
\hline Auswirkung insgesamt & -446 & 459 & 5 & 157 & 802 & 607 \\
\hline
\end{tabular}

Quelle: eigene Berechnungen auf Basis zuvor beschriebener Annahmen.

Allerdings sind hiermit erhebliche fiskalische Folgewirkungen verbunden, die zu ausgesprochen problematischen Ergebnissen führen. Während der Großteil der kommunalen Steuermehreinnahmen auf ebenjener Ebene verbleibt, bezahlt der Landeshaushalt in dem hier analysierten Fall die neu gewonnene Finanzstärke mit Einbußen in hoher dreistelliger Millionenhöhe. Dass hiervon vor allem die anderen Länder und der Bund profitieren, ist allerdings systemimmanent. Neben dem hohen Ausgleichsgrad des FKA trägt auch die spezifische Ausgestaltung der vertikalen Transfers nicht unwesentlich dazu bei.

Dies gilt insbesondere für die sogenannten SonderbedarfsBEZ, vor allem für die BEZ zum Ausgleich unterproportionaler Mittel aus der Forschungsförderung nach $\S 11$ Abs. 6 FAG. Diese Ergänzungszuweisungen, deren Begründung in der Literatur bereits heftig hinterfragt wurde und die als ein Fremdkörper im Gefüge des Finanzausgleichs angesehen werden (Korioth, 2016; Scherf, 2020), führen aufgrund ihrer Konstruktion zu einem Guillotine-artigen Effekt. Sobald ein Land die Grenze der Leistungsschwäche, also eine Finanzkraft von 99,75\%, erreicht oder sogar finanzstark wird, fallen diese Zuweisungen schlagartig weg. Im Falle von RheinlandPfalz hat dies einen Verlust von Zuweisungen in Höhe von 77,5 Mio. Euro zur Folge. Der Effekt kann durchaus auch in die entgegengesetzte Richtung wirken. So scheint es angesichts der aktuellen Finanzkraftentwicklung nicht unwahrscheinlich, dass auch Nordrhein-Westfalen, das nach der vorliegenden Abrechnung des BMF 2021 auf eine Finanzkraft von 99,9\% nach FKA abgerutscht ist, in absehbarer Zeit ein Anrecht auf diese Transfers des Bundes haben wird. 
Auch ohne den ausdrücklichen Verweis auf die Leistungsschwäche als Anspruchsvoraussetzung dürfte der Bezug einer weiteren BEZ zumindest auf längere Frist fraglich sein. Ob Rheinland-Pfalz weiterhin, wie in $\S 11$ Abs. 4 festgelegt, BEZ wegen Kosten politischer Führung in Höhe von 48,3 Mio. Euro erhalten kann, hängt stark von der langfristigen Wirtschafts- und Steuerentwicklung in Mainz ab. Nach der Rechtsprechung des Bundesverfassungsgerichts dürfte es ausgesprochen problematisch sein, einem finanzstarken Land Sonderbedarfs-BEZ zu gewähren, da auch für diese Leistungen „im Regelfall“ gelten muss, dass „die Berücksichtigung von Sonderlasten nicht dazu führen (darf), dass die Finanzkraft des begünstigten Landes die durchschnittliche Finanzkraft der Länder nach dem horizontalen Finanzausgleich übersteigt" (BVerfGE 101, 158, S. 234). Sollte Rheinland-Pfalz aber dauerhaft ein finanzstarkes Land werden, könnte der Bund auch diese BEZ aus verfassungsrechtlichen Gründen infrage stellen.

Am problematischsten erscheinen aber die Konsequenzen von Hebesatzsenkungen in einzelnen Kommunen. Diese können dazu führen, dass der Finanzkraft des jeweiligen Landes höhere Beträge zugerechnet werden, als bei der Gewerbesteuer tatsächlich aufkommt. Diese normierten Einnahmen werden seit der Reform der Bund-LänderFinanzbeziehungen 2020 zu $75 \%$ in den FKA einbezogen - davor zu 64\%. Durch diesen Anstieg nimmt die Wahrscheinlichkeit zu, dass einem Land mehr Steuereinnahmen seiner Kommunen zugerechnet werden, als die Kommunen tatsächlich vereinnahmen. Die kommunalen Entscheidungsträger nehmen ihre Hebesatzautonomie wahr. Sie sind nicht - zumindest nicht unmittelbar - von diesen finanziellen Konsequenzen betroffen, denn die höheren Finanzausgleichsleistungen belasten zunächst allein den Landeshaushalt.

Gelingt es Rheinland-Pfalz (und der Stadt Mainz) jedoch, den Biotechnologie-Standort nachhaltig zu stärken und zusätzliche Arbeitsplätze und Einkommen zu schaffen, könnte das durch die dort generierte Wertschöpfung entstehende Steueraufkommen auf eine breitere Basis gestellt werden. Auf diese Weise könnte ein Teil der hier identifizierten Abschöpfungseffekte der reinen Gewinnbesteuerung und vor allem die negativen Auswirkungen auf den Landeshaushalt gemindert werden.

\section{Literatur}

BMF - Bundesministerium der Finanzen (2022a), Vorläufige Abrechnung des bundesstaatlichen Finanzausgleichs für das Jahr 2021, https:// bundesfinanzministerium.de/Content/DE/Standardartikel/Themen/ Oeffentliche_Finanzen/Foederale_Finanzbeziehungen/Laenderfinanzausgleich/laenderfinanzausgleich.html (wird zeitnah veröffentlicht).

BMF - Bundesministerium der Finanzen (2022b), Kassenmäßige Steuereinnahmen nach Steuerarten und Gebietskörperschaften (aktuelle Ergebnisse), https://bundesfinanzministerium.de/Content/ DE/Standardartikel/Themen/Steuern/Steuerschaetzungen_und_ Steuereinnahmen/1-kassenmaessige-steuereinnahmen-nach-steuerarten-und-gebietskoerperschaften.html (26. Januar 2022).

DIHK - Deutscher Industrie- und Handelskammertag (2021), Hebesätze deutscher Städte und Gemeinden 2021, https://www.dihk.de/de/ themen-und-positionen/wirtschaftspolitik/steuer-und-finanzpolitik/ hebesaetze-56878 (26. Januar 2022).

FM RLP - Ministerium der Finanzen Rheinland-Pfalz (2021), Deutliche Steuermehreinnahmen bei Land und Kommunen - Rheinland-Pfalz wird finanzstark, https://fm.rlp.de/de/presse/detail/news/News/ detail/deutliche-steuermehreinnahmen-bei-land-und-kommunenrheinland-pfalz-wird-finanzstark/ (26. Januar 2022).

Hessenschau (2021), 570 Millionen Euro: Biontech beschert Marburg Geldsegen, https://www.hessenschau.de/wirtschaft/570-millioneneuro-geldsegen-fuer-marburger-haushalt-dank-biontech,marburgbiontech-100.html (26. Januar 2022).

inRLP.de (2021), Geldregen für Mainz und Idar-Oberstein dank Einnahmen an BioNTech-Gewerbesteuer, https://www.inrlp.de/rlp/mainzbiontech-gewerbesteuer-beschert-staedten-ein-plus-im-haushaltart-5330685 (26. Januar 2022).

Korioth, S. (2016), Reform der Finanzbeziehungen von Bund und Ländern: Fairer Kompromiss oder Setzen von Fehlanreizen?, ifo Schnelldienst, 69(24), 5-9.

Mittelhessen (2021), Marburger Koalition zerbricht: Die Linke ist raus, https:// www.mittelhessen.de/lokales/marburg-biedenkopf/marburg/marburger-koalition-zerbricht-die-linke-ist-raus_25014597 (26. Januar 2022).

RND - Redaktionsnetzwerk Deutschland (2021), Steuereinnahmen durch Biontech: Wie Mainz und Idar-Oberstein vom Impfstoffhersteller profitieren, https://www.rnd.de/wirtschaft/steuereinnahmen-durch-biontech-wie-mainz-und-idar-oberstein-vom-impfstoffhersteller-proftieren-SNYMNK4WGSQL5ASSZLK3UJR3YQ.html (26. Januar 2022).

Scherf, W. (2000), Der Länderfinanzausgleich in Deutschland. Ungelöste Probleme und Ansatzpunkte einer Reform.

Scherf, W. (2020), Länderfinanzausgleich 2020: Neue Form - alte Probleme, Wirtschaftsdienst, 100(8), 601-607, https://www.wirtschaftsdienst.eu/inhalt/jahr/2020/heft/8/beitrag/laenderfinanzausgleich2020-neue-form-alte-probleme.html (28. Januar 2022).

Stadtrat Idar-Oberstein (2021), Haushalt 2022, Anlage 1 zu TOP 20.11, https:// idar-oberstein.gremien.info/vorlagen_details.php?vid=20210911100195 (26. Januar 2022).

Statistisches Bundesamt (diverse Jahre), Fachserie 14 Reihe 4, Steuerhaushalt.

SWR - Südwestrundfunk (2021), Unternehmen in Mainz begrüßen Senkung der Gewerbesteuer, https://www.swr.de/swraktuell/rheinlandpfalz/mainz/reaktionen-mainzer-unternehmen-auf-gewerbesteuer-100.html (26. Januar 2022).

The Guardian (2021), Pfizer/BioNTech tax windfalls brings Mainz an early Christmas present, https://www.theguardian.com/world/2021/ dec/27/pfizer-biontech-tax-windfall-brings-mainz-an-early-christmas-present (26. Januar 2022).

Title: Biontech's Tax Booster and Its Impact on Intergovernmental Fiscal Relations

Abstract: The Mainz-based vaccine manufacturer Biontech has had incredible business development in 2021, which has led to a soaring increase in the state of Rhineland-Palatinate's tax revenues. As a result, Rhineland-Palatinate, which was considered financially weak, is now one of the strongest states in Germany and thus is included among the donor states in the financial equalisation system. Such a sharp increase in financial power, however, leads to significant fiscal spillovers that may ultimately result in problematic distributional outcomes. Taking the complex structure of the German fiscal federalism system into account, the authors examine the fiscal consequences of the tax increase from Biontech and come to quite surprising results, especially for the Rhineland-Palatinate state budget. 